It is evident, from the foregoing description, that the Lake Superior fire assay bears no relation to the empirical matting assay in use at Swansea, but is a practical method, easily controlled when once thoroughly learned, and sufficiently accurate for all technical purposes.

\title{
NOTES ON THE ESTIMATION OF COPPER BY POTASSIUN PERMANGANATE.
}

BY H. A. Guess.

Received April 25, rgoz.

Altholgir at various times, during the past few years, different chemists have proposed the use of an alkaline thiocyanate as a precipitant in the estimation of copper, ${ }^{1}$ and although the results have been shown to be sufficiently accurate for technical purposes, the fact remains, that the method has made little headway, in competition with the electrolytic, cyanide, or iodide methods, in technical work, particularly in the metallurgical laboratories of the West, where the iodide method is greatly in favor.

In the iodide method, as generally practiced, the copper is first precipitated by aluminum strips, and while this precipitation is fairly rapid and complete in concentrated solutions, it is not at all so in dilute solutions of 20 milligrams or less of copper in a bulk of $150 \mathrm{cc}$. of dilute sulphuric acid. Furthermore, other metals, particularly arsenic, interfere by being precipitated along with the copper, and have to be rendered inert by oxidation with potassium chlorate. On the other hand the precipitation of copper as thiocyanate is practically complete at the moment of adding the precipitant; no other metals interfere, silver being the only other metal precipitated by an alkaline thiocyanate from an acid solution, and its thiocyanate is unacted upon by the subsequent caustic alkali treatment.

It has seemed to the writer that the main drawback to the method as so far proposed is the use of asbestos as the filtering medium, and the consequent necessity of filtering-pumps, which in a laboratory doing 100 or more copper assays in a day would render the process awkward and cumbersome. For the past six

1 This Journat, 20,610; 22, 685, tt seg; Am. J. Sci., 163, 20-26; Chem. Centrbl., 1, (19), 1085 (1899). 
months the writer has used a modified form of this process, for the estimation of copper in ores, tailings, and concentrates, doing away with asbestos as filtering material, and using filtering paper well extracted (with hydrochloric acid and hydrofluoric acid) instead, the process being briefly as follows :

From I to 5 grams, depending on the material for assay, are digested in an 8-ounce flask with nitric and hydrochloric acids, together with a few drops of sulphuric acid, to get rid of any lead present, the excess of acid is boiled away, the acid solution diluted, filtered, and the residue well washed on the filter. The acid filtrate is neutralized with ammonia, and then rendered just acid by a few drops of dilute hydrochloric acid; the acid cupric solution is reduced by an excess of sodium sulphite, or sulphurous acid previously nearly neutralized by sodium carbonate, and the copper precipitated as white granular cuprous thiocyanate by a slight excess of potassium or ammonium thiocyanate. The mixture is heated to boiling, the precipitate settling rapidly; the clear supernatant liquid is decanted through an II-cm. filter, and the residue, after washing by decantation, is finally washed upon the filter, and thoroughly washed with hot water. The funnel, with its filter and precipitate, is now placed in the original precipitation flask, and a boiling Io per cent. solution of caustic soda poured through. The operation is repeated with a further amount of the caustic solution, the filter being filled full each time to insure complete contact with all of the precipitate. Complete decomposition of the cuprous thiocyanate takes place, the equation being $\mathrm{CuCNS}+\mathrm{NaOH}=\mathrm{CuOH}+\mathrm{NaCNS}$; the insoluble yellow cuprous hydroxide remains on the filter, the alkaline thiocyanate passing through. The filter and contents are well washed with hot water, the alkaline filtrate made decidedly acid with dilute sulphuric acid, and the liberated thiocyanic acid titrated with standardized potassium permanganate at the warm temperature given to it by the hot water washings.

This method, like most other technical ones, is not perfect. The chief imperfection lies in the slight solubility of cuprous thiocyanate in the solutions present. Careful experiments as to this solubility have been made by testing the filtrate and washings from the precipitation, as thiocyanate, of weighed amounts of pure copper; the copper in solution in the filtrate is determined colori- 
metrically by potassium ferrocyanide, and also by formaldoxime ${ }^{1}$ (prepared by mixing 20 per cent. formaldehyde solution with an equimolecular proportion of hydroxylamine hydrochloride), the reaction being an intense violet coloration for the faintest traces of copper. It was found in this way that the most complete precipitation of the copper was obtained when made under the conditions above outlined, and even under these conditions the precipitate is to a slight extent soluble, the degree of solubility being most accurately determined by standardizing potassium permanganate solution with pure copper as above described, and comparing the value thus obtained with the copper value calculated from the carefully determined iron value of the same solution. Thus, comparing the equation

$$
\begin{aligned}
\mathrm{IOHCNS}+12 \mathrm{KMnO}_{4}+8 \mathrm{H}_{2} \mathrm{SO}_{4}= \\
6 \mathrm{~K}_{2} \mathrm{SO}_{4}+\mathrm{I}_{2} \mathrm{MnSO}_{4}+\mathrm{IOHCN}+8 \mathrm{H}_{2} \mathrm{O}
\end{aligned}
$$

for thiocyanic acid and permanganate with the iron equation for permanganate, the copper-iron ratio is $10 \mathrm{Cu}: 60 \mathrm{Fe}$ and the factor for copper from the iron value is 0.1892 . The copper value, as determined by the precipitation (as thiocyanate) and titration, represents a factor of approximately 0.192 of the iron value, the difference between the two being due to the slight solubility of the cuprous thiocyanate and the consequent incomplete precipitation.

By standardizing the permanganate solution always against pure copper, under the same conditions as the regular assays, this error of solubility is for all technical purposes eliminated. The other imperfection of the method is the action of the filter-paper on the hot caustic alkali solution, which is very slight, and is constant for the same type of filter. It can be ascertained by running a blank filter under similar treatment, titrating, and making the correction. On an II-cm. filter, it amounts usually to $0.3 \mathrm{cc}$. potassium permanganate, of a strength of I cc. equal to 2 milligrams copper.

The method has been run in parallel with the iodide on the same samples in this laboratory during the past six months, in daily assays of concentration-mill products, and in 'control' assays on smelter shipments, some 3000 determinations having been made by the method in that time. The method was begun in

1 A. Bach: Compt. rend., 128, (6), 363-365. 
a tentative way at first for tailings assays, for which neither the cyanide nor iodide methods were suitable, nor, from the arsenic and manganese present, was the usual colorimetric method. It was later adopted for the assays on crude ore and concentrates, and its results have been uniformly satisfactory. In practice it takes about the same time as the iodide method, and while, for copper to the amount of 3 per cent. and upwards, it may not offer many advantages over the latter method, which cannot well be improved upon, it is more accurate than, and distinctly superior to, either the iodide or cyanide method for small or fractional percentages of copper.

LABURATORY OF THE SILVER LAKE MINES,

Silverton, Colo., April Ig, IgOz.

[CONTRIBUTION FROM THE LABORATORY OF THE BUREAU OF INTERNAL Revenue, U. S. Treasury DhPartment.]

\section{THE INFLUENCE OF THE GROWTH OF MOLD UPON THE CHEMICAL COMPOSITION OF OLEOMAR. GARINE AND BUTTER.}

By Charles A. Crampton.

Received April 12, 2902.

Havine occasion to pass through the ordinary course of analysis a number of samples of oleomargarine, which had been kept in glass-stoppered bottles for about two years, I was much surprised at the results obtained. The samples, about fifty in number, had a well-authenticated history of having been taken from different packages of the same shipment of oleomargarine, a shipment which was represented by a single sample taken at the time and examined in this laboratory, giving a normal Reichert-Meissl figure.

Instead of obtaining the usual figures from the fifty old samples, as would be expected, and a close agreement among themselves, the results varied greatly; and what was still more striking, the figures given by one determination-that of the refractive indexwere so low in some cases that the samples would have been classed as butter, if judged by this test alone, while others gave results normal for oleomargarine. Many of the samples were permeated with a growth of dark green mold, and on tabulating the results, it was soon seen that this was the cause of the differences, the 